\title{
ALGUNAS CONSIDERACIONES SOBRE LOS MONUMENTOS 75 Y 80 DE LA VENTA, TABASCO
}

REBECCA B. GONZÁlEZ LAUCK

Entre los resultados de los trabajos realizados por el Proyecto Arqueológico La Venta, patrocinado por el Instituto de Cultura de Tabasco y el Instituto Nacional de Antropología e Historia, está el haber incorporado al corpus escultórico de La Venta -ciudad principal y prominente de la civilización olmeca- diez fragmentos $y / o$ esculturas completas labradas en piedra anteriormente desconocidas. Puesto que todas ellas serán objeto de publicación en un futuro cercano, se pretende proporcionar en el interim una descripción más completa de lo que hasta ahora existe publicado sobre dos de ellas: los monumentos 75 y 80 .

El corpus escultórico olmeca es relativamente extenso y se define por un estilo particular (de la Fuente 1973, 1977). Se identifica en términos formales por su preocupación por el volumen, sus formas cerradas y un refinado sentido de proporción, lo cual impregna las piezas de todo tamaño con una impresión de monumentalidad (de la Fuente Ibid, Graham 1981, 1989). Tres categorias básicas caracterizan el corpus de escultura monumental olmeca: las figuras humanas, de animales y de las compuestas. Dentro de estas categorías es posible identificar ciertos temas iconográficos y sus variaciones (de la Fuente: 1977). Los monumentos 75 y 80 de La Venta son dos ejemplos completos dentro de una misma temática -la de seres sobrenaturalesque por su buen estado de conservación, nos permiten una mejor definición de los mismos.

El monumento 75 se encontraba hasta hace poco incompleto. El fragmento inferior del monumento 75 (fig. 1) es conocido desde hace más de quince años (de la Fuente 1973: 119). Su ubicación original dentro de la traza arquitectónica del sitio la presenta Clewlow (1974: fig. 6), pero por desconocimiento del catálogo de B. de la Fuente (1973) lo nombra "monumento 74". En el más reciente plano de la zona arqueológica (González Lauck 1988: fig. 1), el monumento 75 queda ubicado aproximadamente sobre la orilla oeste de la "Acrópolis" Stirling donde apareció en 1969 cuando se excavaron los caminos que entrecruzan esta inmensa construcción (Clewlow 1974: 40). Esta pieza, 
como la gran mayoría de las esculturas de La Venta, fue trasladada al Parque Museo La Venta y subsecuentemente a la Sala de Piezas Monumentales en la planta baja del Museo Regional de Antropología-Carlos Pellicer Cámara en Villahermosa, donde se encuentra hoy en día. El fragmento superior del monumento 75 no ha sido publicado con anterioridad (fig. 2). Aproximadamente desde 1984, se encontraba almacenado en la Delegación Municipal de Villa La Venta y a finales de 1988 fue trasladado al recién creado Museo Arqueológico La Venta para su exhibición.

Entre los programas de trabajo del Proyecto Arqueológico La Venta se realizaron reproducciones en resina de la mayoría de las esculturas procedentes de La Venta que se encuentran en Villahermosa, con la finalidad de integrar las copias al conjunto arquitectónico del sitio. Es así que se realizó la reproducción de la parte inferior del monumento 75 y se trasladó a La Venta donde, en vez de ubicarla en la zona arqueológica como originalmente se había programado, se incorporó a la museografía del museo de sitio. Fue gracias al excelente sentido de observación del Sr. Roberto Jiménez Alvarez, custodio de la zona arqueológica, quien detectó que la copia de resina de la parte inferior del monumento 75 formaba una escultura completa al unirse con el anteriormente mencionado fragmento.

El monumento 75 (fig. 3), es una escultura en bulto con detalles en alto y bajo relieve. Representa una figura humana de pie, utilizando una placa bucal. ${ }^{1}$ La escultura fue labrada en basalto (Clewlow 1974:

1 Resulta interesante que son pocos los investigadores que identifican el uso de placas bucales en el arte olmeca como tal. En el compendio de símbolos olmecas de Joralemon (1971), no se logra establecer la diferencia entre lo que es la boca de las diversas figuras y las diferentes placas bucales, sino que todo se interpreta como algo monolítico y se les conoce popularmente como "classic Olmec mouth with flaring upper lip and drooping lower corners" (op. cit: 9) o el "typical jaguar-god mouth cluster" (Clewlow 1974: 40), para mencionar sólo un par de ejemplos. Sin embargo, parece que no es tan fantástico el asunto, aunque seguramente dichas placas son elementos que le confiere al personaje representado ciertas características simbólicas.

Dichas bocas, en realidad parecen estar conformadas por dos diferentes componentes; a) los labios y b) las placas bucales. Estas últimas, parecen tener diferentes formas pero, por lo general, son elementos que cubren el espacio entre las fosas nasales y el labio superior, al mismo tiempo que jala hacia arriba y esconde éste último bajo la placa, exponiendo las encías. Por lo común, éstas son representadas carentes de dientes o con dos o cuatro colmillos, que muy probablemente formen parte de las mismas placas. Se cree que las placas bucales son de dos tipos: 1) las que cubren solamente la parte superior de la boca y 2) las que encubren toda el área bucal. Sin embargo, cabe señalar que, en algunos casos se representan sencillamente los labios de la figura representada redoblados hacia arriba, en forma de un animal (ya sea felino, canino, etc.) en proceso de grunir. 
40) y completa mide 1.10 metros de alto, por 37 centímetros de ancho y 48 centímetros de espesor. Se le da mayor énfasis a la cabeza de la figura, que al resto del cuerpo. Esto no es sólo en términos de proporción sino que también en los detalles de la fisonomía. En esta figura la cabeza y su casco conforman más de la mitad de la escultura, mientras que el cuerpo está representado en forma reducida y esquemática. La pieza es un prisma rectangular levemente más angosto hacia su parte superior, sobre el cual se redondearon algunas esquinas y se eliminaron ciertas partes de la piedra para definir el volumen compacto. Es una forma cerrada, autocontenida completamente y descrita como "rígida" (de la Fuente 1984: 181).

La cabeza de la figura, un volumen rectangular con esquinas redondeadas, es la de un ser humano. El rostro está enmarcado por un casco y sus ataduras. Los ojos son grandes y ovalados y están labrados en bajorrelieve. La nariz es delgada en su puente, pero ancha y achatada a la altura de las fosas. Pegada a éstas se encuentra una placa bucal trapezoidal (cuya orilla superior es recta y está pegada a la nariz, mientras que sus lados se extienden hacia la parte inferior de la misma, que tiene una forma curva), cubriendo el espacio entre la nariz y el labio superior. La placa bucal jala el labio superior debajo de la misma, exponiendo las encías de la boca entrebierta del individuo. El labio inferior tiene las comisuras hacia abajo y enmarca parcialmente el prominente mentón.

El casco, se asienta hasta la altura de las cejas del individuo, o sea, cubre toda la frente y está delimitado por una banda en su borde inferior que circunda toda la cabeza. En su parte central al frente se encuentra una banda realzada que se extiende hasta alcanzar la banda inferior. Al centro del casco se entrecruzan dos bandas delgadas, que se extienden hacia sus cuatro esquinas, aunque no se puede apreciar en su totalidad puesto que el relieve ha desaparecido en algunas par-

Es probable que el estudio sistemático de estas placas bucales nos brinde respuestas que nos permitan un acercamiento hacia una mejor comprensión de su función, naturaleza y significado, además como posibles marcadores temporales. Pohorilenko (1977) las agrupa como "referential signs", a la vez que Proskouriakoff (1979: 113) nos señala que las caras grotescas o formas zoomorfas compuestas en el arte olmeca y maya pueden ser representaciones de seres supernaturales en vez de verlas como una composición de símbolos.

La existencia de placas bucales en otras culturas y tiempos de la historia antigua del Nuevo Mundo es conocida. El no tener un ejemplo que se pueda ligar directamente a la civilización olmeca, quizá sea resultado de haber sido elaboradas en materiales perecederos, como el cuero. 
tes. Debajo del borde inferior cuelgan dos bandas verticales que enmarcan por ambos lados el rostro de la figura. Estas bandas terminan a la altura de la parte posterior de la quijada y detrás de ellas están representadas, muy sencillamente y en bajorrelieve, las orejas del personaje.

La parte inferior de la escultura representa el cuerpo de la figura, el cual se une a la cabeza, sin un cuello visible. En esta parte de la figura todos los elementos se reducen casi a su mínima expresión y adquieren una configuración de bloque y geométrica. Los brazos de la figura se encuentran representados flexionados en ángulo recto con las manos extendidas y las palmas hacia dentro. Da la impresión de que con ellas sostiene un objeto rectancular - ¿una placa? - que cubre casi todo lo que fue el frente del torso de la figura. Esta placa tiene una línea incisa que va paralela al borde, como un margen interior. Tal vez dentro de este espacio existía un diseño labrado, pero ahora sólo se pueden apreciar algunas líneas incisas, sin arreglo particular.

Las piernas están remetidas un poco debajo de la placa y señaladas por una línea incisa profunda. Los pies están representados como una superficie plana e inclinada que se extiende de las piernas. Los dedos del pie aparecen señalados sencillamente por incisiones. Estos descansan sobre un pequeño pedestal que funciona como base para la escultura.

En cuanto a la parte posterior el monumento 75 tiene como único detalle decorativo, la banda inferior del casco. Debajo de ella, la superficie está muy bien tallada, hasta dejarla casi plana y sin irregularidades; se advierte un tratamiento similar en algunas de las cabezas colosales, lo cual ha sido explicado recientemente como residuo de su forma anterior (Porter 1989).

El monumento 75 de La Venta, ha sido comparado con la escultura de Ojo de Agua, Chiapas (Navarrete 1974), puesto que Milbraith (1976: 26) considera que representan "temas casi idénticos". Asimismo, se ha comparado con el Monumento 52 de San Lorenzo (Clewlow 1974: 40, de la Fuente 1977: 181), por similitudes formales y de ciertos elementos tales como: las placas bucales. En realidad, es difícil encontrar piezas olmecas que correspondan exactamente, pues hay una gran variación dentro de los diversos temas representados que quizá son un reflejo de diferencias temporales o regionales. Una pequeña figura de jadeita, de procedencia desconocida, en la colección de Dumbarton Oaks (Benson 1981: fig. 5) es muy similar en formato al monumento 75. La principal diferencia entre estas piezas es que una es portátil, mientras la otra difícilmente se le puede considerar así. Es interesante, 
sin embargo, que el mismo tema sea repetido en piezas portátiles, al igual que en esculturas monumentales. Cabe señalar, que hay diferencias en la posición de las manos y en el casco, las cuales pueden ser importantes en la comprensión de su significado. El hacha votiva de la Casa de la Cultura de Juchitán, Oaxaca (Pohorilenko 1982: fig. 2) también parece representar el mismo tema, aunque presenta aún más diferencias en la forma de representación, quizá como reflejo de sus distintas funciones.

Por otro lado, el monumento 80 de La Venta (figs. 4 y 5 ), forma parte de una temática diferente dentro de la tradición escultórica olmeca. Este representa a un animal sentado (González Lauck 1988: 154), tema que es repetido con ciertas diferencias en dos esculturas conservadas incompletas: el monumento 1 de Los Soldados (fig. 6) (Medellín Zenil 1961: 11), sitio ubicado río abajo de La Venta sobre el Tonalá, y el monumento 37 de San Lorenzo (fig. 7) (Coe and Diehl 1980: 346), también en Veracruz. Puesto que el monumento 80 es el ejemplo mejor conservado de los tres, a través de él se logra una mejor apreciación de las otras dos esculturas.

Aunque ya se presentó una descripción general del monumento 80 de La Venta (González Lauck Ibid), se aprovecha la ocasión para corregir errores y omisiones previos. Dicha pieza es una escultura en bulto representando a un felino en posición sedente, con las patas posteriores dobladas en ángulo y terminando en vez de zarpas, en pequeñas cabezas de serpientes (J. Porter, comunicación personal). Esto se puede apreciar mejor en la pata derecha de la figura, puesto que la izquierda no está bien conservada. El animal está ligeramente inclinado hacia el frente, descansando sobre sus patas delanteras, las cuales aparecen en posición recta y firmes. La terminación de estas patas están destruidas, razón por la cual no se puede apreciar si estaban decoradas de alguna forma especial.

En comparación, las patas delanteras y traseras del ejemplar de San Lorenzo están representadas en posición similar a las del monumento 80 de La Venta, excepto que en su terminación tienen forma de garras estilizadas, como las de un felino. En cuanto a las extremidades de la figura representada en el monumento 1 de Los Soldados éstas muestran mayor divergencia. La posición de las extremidades posteriores de esta figura, no es muy clara, pero parecen corresponder a las de un ser humano sentado con una pierna cruzada y la otra doblada debajo de sí misma; posición imposible para un felino, pero similar a algunas de las figuras humanas sedentes olmecas. Las extremidades 
delanteras, al igual que en los otros dos monumentos, se extienden y sostienen el cuerpo de la figura. Las "manos" están representadas simplemente con unas líneas incisas para señalar los dedos, tratamiento similar que se les da a otras figuras humanas en estilo olmeca. Por estas características se ha descrito a este felino como "antropomorfo" (Medellín Zenil 1971: 22).

El animal representado en el monumento 80 de La Venta parece estar en una posición de descanso atento; su espalda es recta y no presenta la curvatura, ni la inclinación que se aprecia en el monumento 1 de Los Soldados y en el monumento 37 de San Lorenzo. La cabeza del animal es casi cuadrada, si se ve de frente, mientras que de perfil es subrectangular, lo cual rompe con la silueta de "pirámide trunca" (de la Fuente 1977: 308) que exhiben los cuerpos de estos tres animales.

El rostro del felino de La Venta, está compuesto por una serie de volúmenes que le dan una calidad de pesantez. Los ojos están señalados por una hendidura entre la masa carnosa de las mejillas y la frente prominente. El hocico es ancho. Las fosas nasales no se distinguen, en contraste con las del monumento 1 de Los Soldados, al igual que dicho monumento, éste presenta un elemento en forma de triángulo invertido que cuelga de la parte superior y central de la boca entreabierta. ${ }^{2}$ Por atrás de dicho elemento cuelga una banda cilíndrica que cae, en dos partes, entre las patas delanteras de cada uno de los tres animales.

Este elemento en forma de banda cilíndrica ha sido interpretado en la escultura de San Lorenzo y por extensión a la pieza de Los Soldados, como una "cuerda atada a manera de collar" (Beverido 1970: 121), "largos colmillos (?)" (de la Fuente 1973: 161) o "tusklike fangs" (Coe y Diehl 1981: 344). Afortunadamente, este elemento se encuentra bas-

2 Continuando con el tema de placas bucales olmecas de la nota anterior, es importante no sólo identificarlas claramente, sino también distinguir los diferentes elementos que las componen. La representación de un triángulo invertido colgante de la parte central y superior del área bucal en figuras olmecas es un rasgo poco frecuente. Este se presenta en los monumentos 80 de La Venta, i de Los Soldados, 1 de Laguna de Los Cerros, un monumento de procedencia desconocida en el Museo de Santiago Tuxtla (pieza 203 en el Catálogo de Escultura Monumental Olmeca) y el "tigrillo" de Matacanela. En ésta última escultura, Castro Leal (1982: 51) ha identificado este elemento como lengua. En las piezas de Laguna de los Cerros y en el Museo de Santiago Tuxtla, dicho elemento parece formar parte de la placa bucal. Joralemon (1971: 43) describe el área bucal de la escultura de Laguna de los Cerros como "fang-filled mouth", sin diferenciar los diferentes componentes. En contraste, en lós casos de las esculturas de La Venta y Los Soldados, parecen estar incorporados al hocico del animal representado. Al referirse a la escultura de Los Soldados, Coe y Diehl (1980: 347) describen este elemento como un "egg-tooth". 
tante bien conservado en el monumento 80 de La Venta, lo cual permite identificar que lo que cae de la boca del felino es una serpiente.

El cuerpo del ser viperino está decorado con una serie de líneas diagonales incisas sobre la piedra, las cuales también se pueden apreciar en la pieza de Los Soldados, pero no en la escultura de San Lorenzo puesto que dicho elemento está muy destruido en esta pieza. En el monumento 80 , la cabeza de la serpiente (fig. 8) está representada verticalmente en bajorrelieve, en la parte terminal de la banda izquierda, la cual se amplía levemente y cubre cerca de la tercera parte de la misma. Está dividida en dos espacios verticales, y en uno se puede apreciar su ojo grande y ovalado al igual que las fosas nasales de la serpiente, mientras, que en el otro, se representa la boca entreabierta de la serpiente con su(s?) colmillo(s?).

En el extremo opuesto del cuerpo de serpiente, o sea en la parte terminal de la banda derecha, también se encuentra labrada en bajorrelieve. Anteriormente este diseño se interpretó como otra cabeza de serpiente, sin embargo, después de un examen cuidadoso, se piensa que representa la cola del animal (fig. 9). Si se hubiese querido representar otra cabeza de serpiente, sería lógico que fuese representada igual a la del otro extremo, lo cual no se hizo. Este extremo está dividido básicamente en dos espacios verticales: en el espacio "interior" se encuentran tres rectángulos redondeados con una depresión hacia su centro, enmarcados por una banda con esquinas redondeadas, mientras que en el espacio "exterior" existe una banda curvilínea en relieve hundido y un elemento como gancho en su punta, en bajorrelieve; elementos estilizados que quizá identifican específicamente cierta especie de serpiente.

El elemento que cuelga de la boca de la escultura de Los Soldados, parece ser una versión más burda y estilizada de la serpiente del monumento 80 de La Venta. El cuerpo presenta, como ya se mencionó, las líneas incisas diagonales, pero en vez de las seis que se pueden apreciar en la pieza de La Venta, sólo se marcaron tres en cada lado. Los elementos terminales (la cabeza y cola) del ser vípero en la escultura de Los Soldados presenta poco interés en los detalles pero enfatiza su importancia por el desproporcionado espacio que se les confiere. En la pieza de La Venta, la cola y la cabeza de la serpiente cubren aproximadamente un tercio de cada columna, mientras que en la pieza de Los Soldados éstos ocupan casi la mitad. Sin embargo, la representación de la cabeza y la cola es extremadamente esquemática, puesto que no se aprecian los detalles como en el monumento 80. 
Los elementos terminales del monumento 1 de Los Soldados, están separados del cuerpo viperino por unas bandas horizontales, como si fuesen anillos gruesos. La cabeza de la serpiente está indicada simplemente por unas líneas incisas, como si fuese un esbozo, respetando los espacios que corresponden al ojo, nariz, mandibula y colmillo. La cola también se representa en dos espacios verticales separados por una línea burda incisa. Se encuentra, además, en este espacio una banda delgada en forma de anillo, en bajorrelieve. No se aprecia ningún elemento similar en el monumento 80 de La Venta.

Aunque, como ya se ha visto, los elementos colgantes de la escultura de San Lorenzo han sido los que han estado sujetos a diversas intepretaciones, son los que por su pésimo estado de conservación, presentan el menor número de elementos para su correcta definición. La banda izquierda está completamente destruida, excepto por una pequeña sección en su punta. Ahí se puede distinguir una línea incisa que tal vez corresponda a la línea que divide las fosas nasales de la mandibula de la serpiente, si es que tuviese el mismo tratamiento que la escultura de Los Soldados. La banda derecha se encuentra menos destruida y se puede apreciar casi en su totalidad la terminación de la misma, la cual está delimitada en su parte superior por una banda horizontal en bajorrelieve. La banda o anillo está dividida en dos partes horizontales y parece que la superior tiene líneas diagonales incisas como decoración. Debajo de la banda, el espacio está dividido en dos partes verticales y también se puede apreciar la misma separación horizontal que se ve en la pieza de Los Soldados. Cabe señalar, que la autora hace la descripción de este monumento con base a las fotografías publicadas puesto que nunca ha visto esta escultura, razón por la cual puede incurrir en más errores de lo común.

En relación a los diferentes tamaños de las esculturas, en su actual estado, el monumento 1 de Los Soldados es el más grande de los tres. Si estuviera completo se piensa que fácilmente podría medir unos 40 50 centímetros más que el monumento 80 de La Venta. El monumento 37 de San Lorenzo es el más pequeño de los tres, midiendo el fragmento que se conserva únicamente 60 centímetros.

Se tiene poca información en cuanto al contexto arqueológico de estas tres esculturas, aunque se reconoce que éste no necesariamente coincide con la fecha de elaboración de las esculturas. El monumento 80 fue excavado sin supervisión técnica -por lo cual sólo se tiene la ubicación superficial original- cerca de la esquina noroeste de la estructura A-5 de La Venta. Para el monumento 1 de Los Soldados tam- 
DOI: http://dx.doi.org/10.22201/iie.18703062e.1991.62.1586

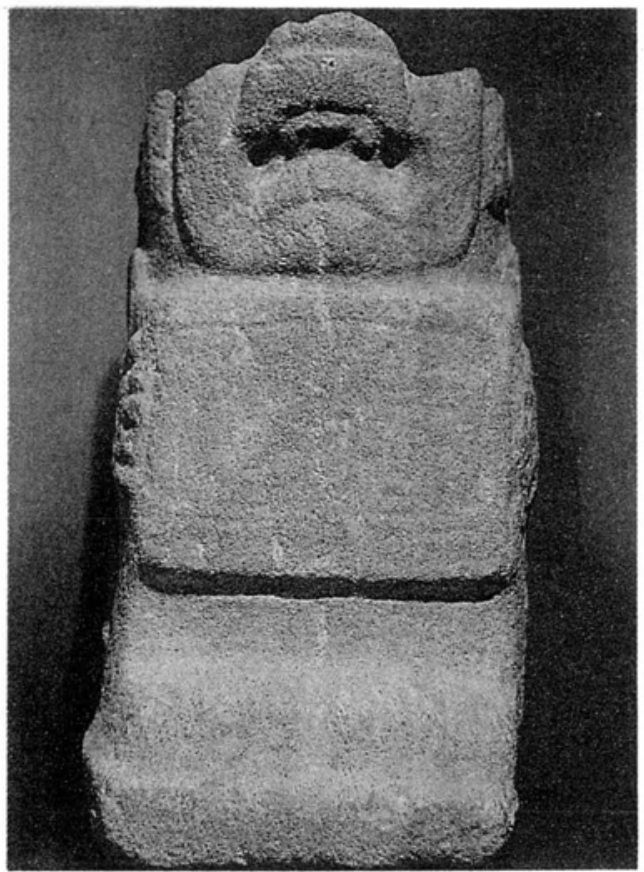

1. Fragmento inferior del Monumento 75 de La Venta. Museo Regional de Antropología, Villahermosa, Tabasco.

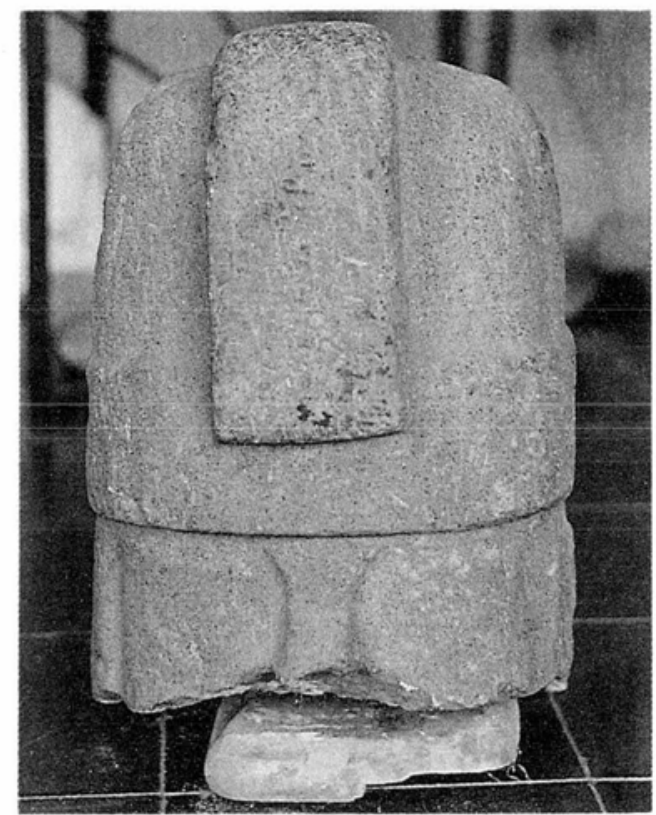

2. Fragmento superior del Monumento 75 de La Venta. Museo de Sitio en Villa La Venta, Huimanguillo, Tabasco. 


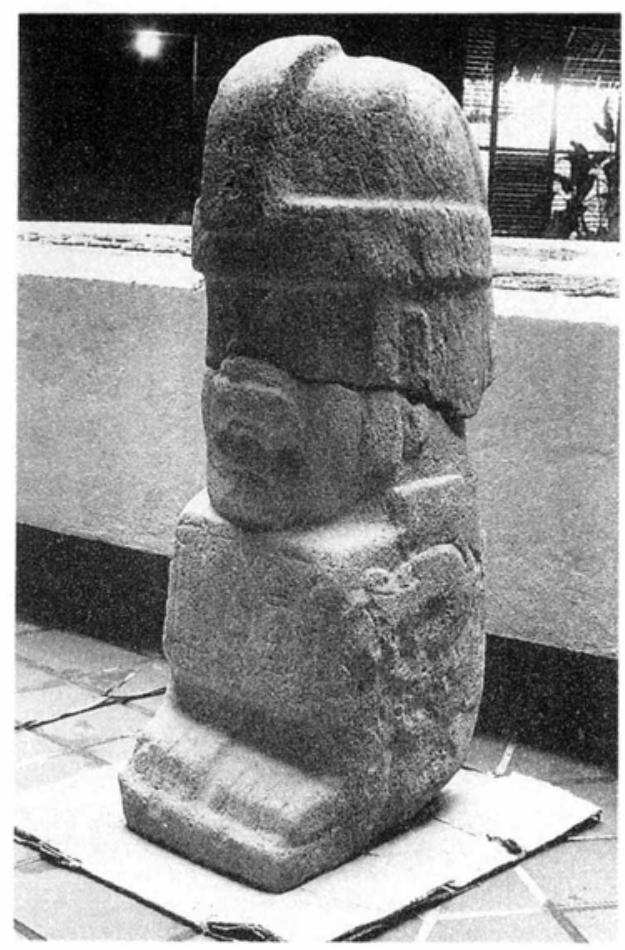

3. Monumento 75 (fragmento inferior, es una reproducción del original). Museo de Sitio en Villa La Venta, Huimanguillo, Tabasco.

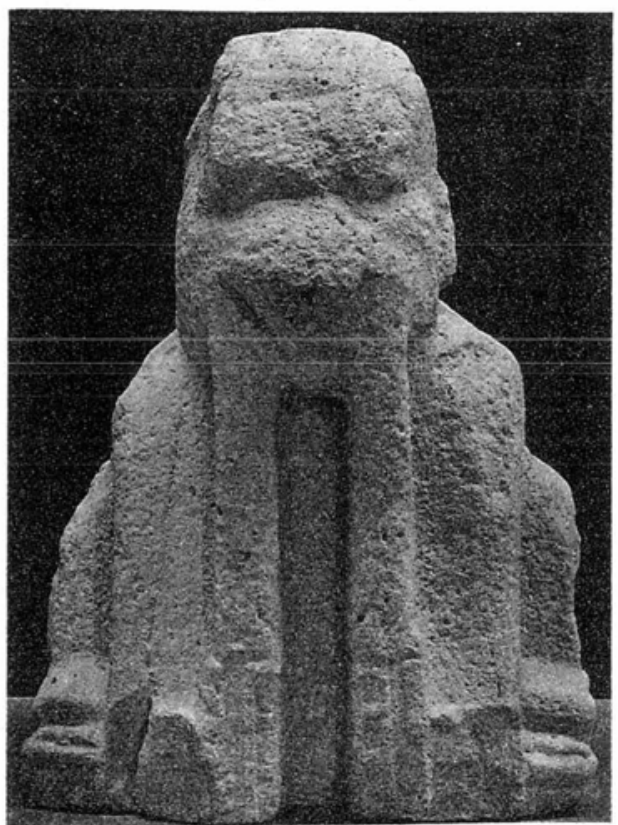

4. Monumento 80 de La Venta. Museo de Sitio en Villa La Venta, Huimanguillo, Tabasco. 
DOI: http://dx.doi.org/10.22201/iie.18703062e.1991.62.1586

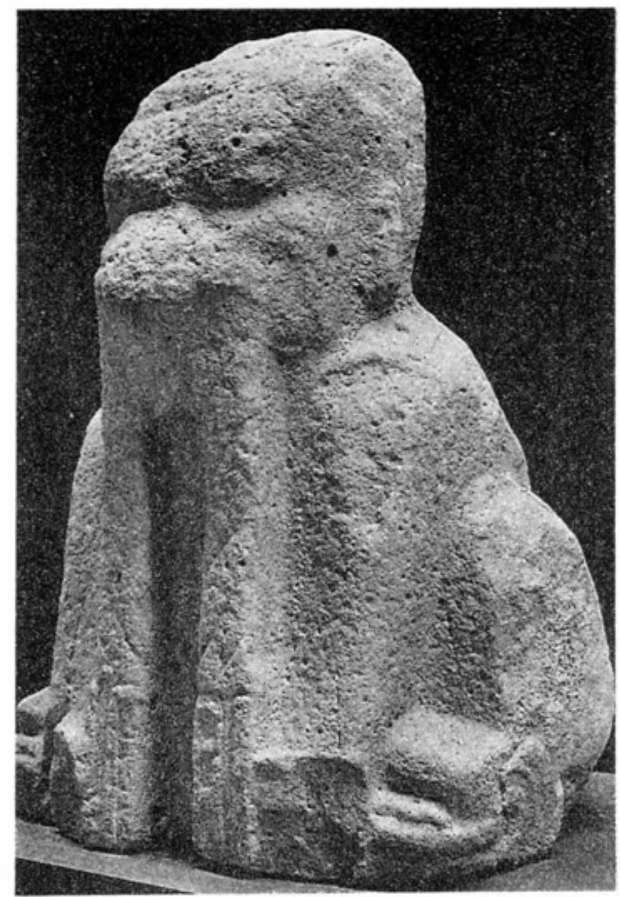

5. Monumento 80 de La Venta. Museo de Sitio en Villa La Venta, Huimanguillo, Tabasco.

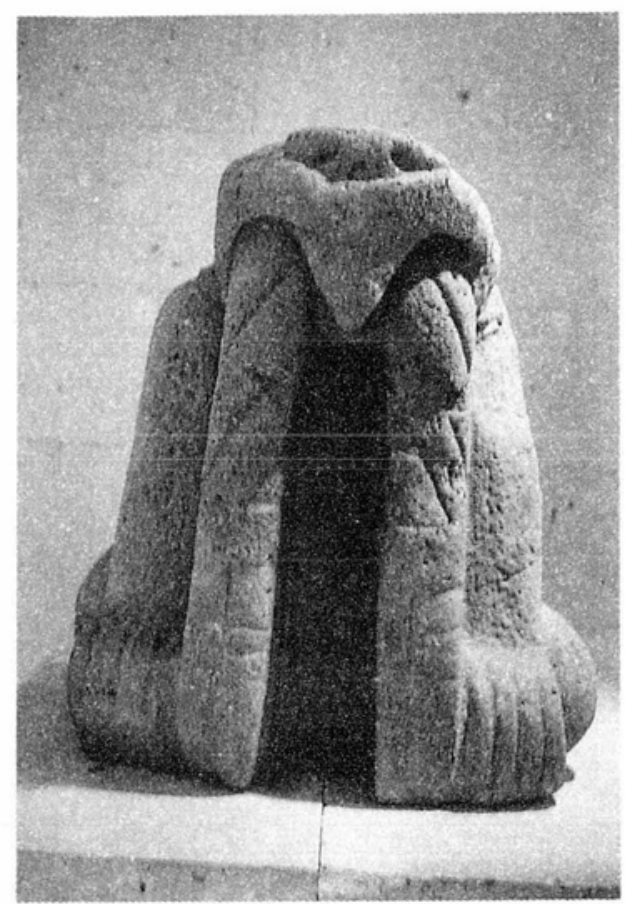

6. Monumento 1 de Los Soldados, Veracruz. Museo de Antropología, Xalapa, Veracruz. 


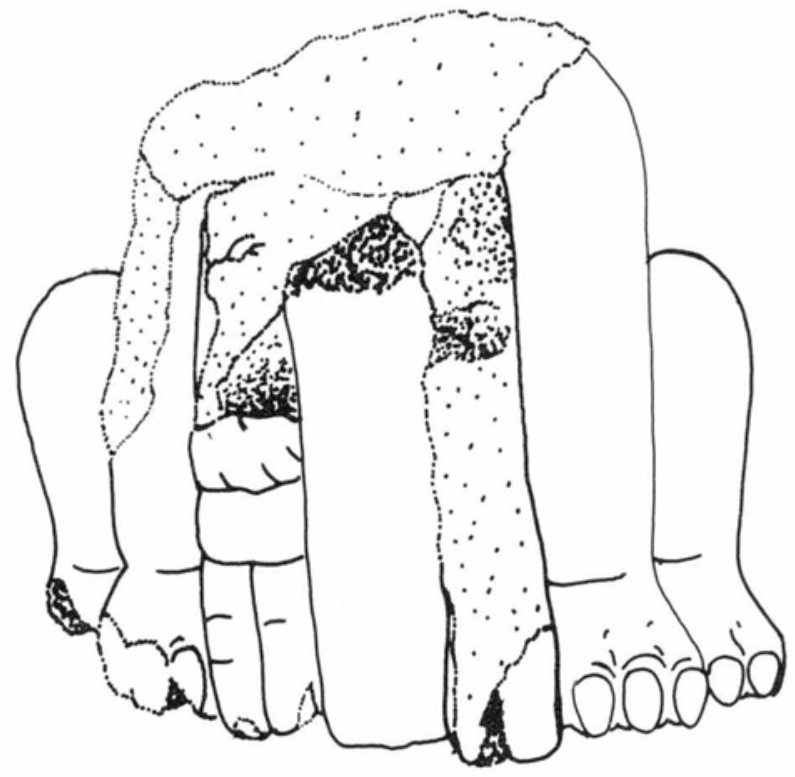

7. Monumento 37, San Lorenzo, Veracruz. (Tomado de Milbraith, 1979:59) Dibujo: Isabel Rabadán.

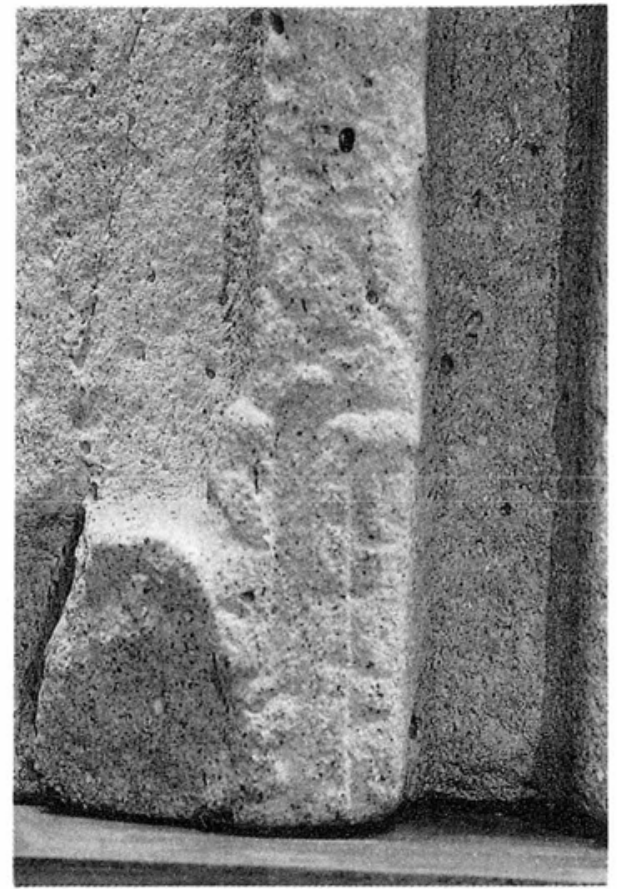

8. Detalle de la cabeza de la serpiente del Monumento 80 de La Venta. Museo de Sitio en Villa La Venta, Huimanguillo, Tabasco.

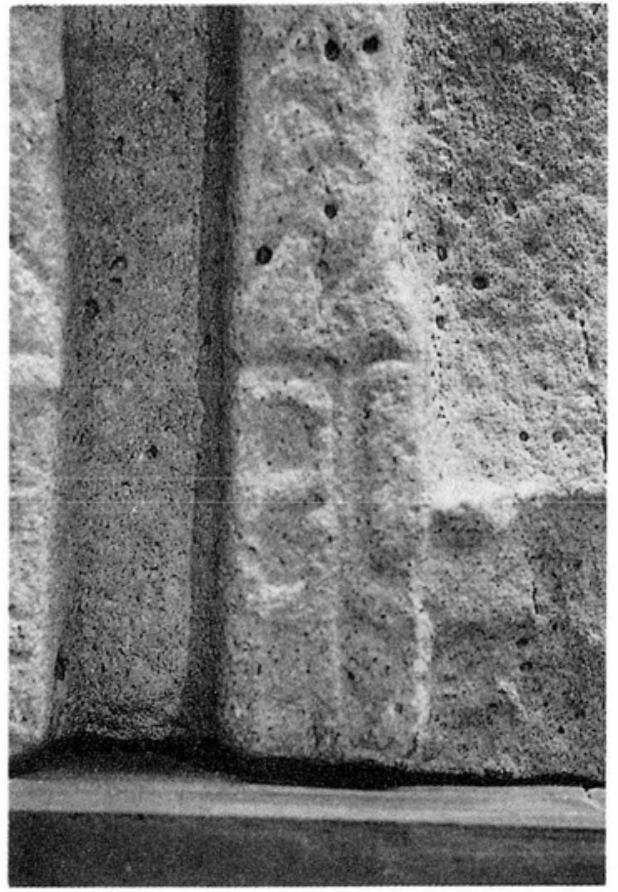

9. Detalle de la cabeza de la serpiente del Monumento 80 de La Venta. Museo de Sitio en Villa La Venta, Huimanguillo, Tabasco. 
poco existe información estratigráfica. El monumento 37 fue encontrado sobre la loma del Grupo D de San Lorenzo, donde estaba alineado con otros seis monumentos asentados directamente sobre el estrato $\mathrm{D}$ el cual, con base en la secuencia cerámica del sitio, corresponde a la fase San Lorenzo A y B (1150-900 a.C.) (Coe y Diehl 1980: $111-116$ y 346 ).

Suponiendo que estas esculturas tratan la misma temática, se podría sugerir que son un reflejo de los cambios temporales y espaciales que se han identificado previamente por B. de la Fuente (1977: 90) en el corpus escultórico olmeca de la Costa del Golfo. Aún en su estado fragmentario, el monumento 37 de San Lorenzo parece ser el ejemplo más "clásico olmeca" en estilo de los tres, por la pureza en su forma y contenido. El monumento 80 de La Venta, a su vez, es un reflejo del estilo propio de La Venta donde se incorporan ciertos cambios (iun mayor simbolismo?), pero manteniendo la estructura básica que se puede apreciar en la pieza de San Lorenzo. Por otro lado, la escultura de Los Soldados, parece reflejar el estado secundario o provincial, quizá hasta temporalmente más tardío, del sitio. Lo que no podían imitar los escultores de Los Soldados en forma y contenido, lo trataron de reemplazar con un mayor tamaño de piedra. Por falta de calidad artística o quizá un cambio en el sistema simbólico o por falta de una adecuada comprensión del tema representado en las otras dos esculturas, los rasgos de mayor importancia (i.e. el cuerpo de felino, la representación de la serpiente colgante) son interpretados de forma diferente o incorporando nuevos elementos o no se les da la importancia que presentan en las otras esculturas.

También existen otras dos esculturas que presentan similitud, pero a un menor grado, con el monumento 80 de La Venta. Una de ellas es una pequeño pieza procedente de Matacanela, Veracruz (Castro Leal 1982). Esta pieza también representa un animal sedente, que ha sido interpretado como "tigrillo" o "pequeño tigre" (Ibid). En el dibujo de la pieza, aunque no es tan claro en la fotografía, se puede apreciar que de su hocico emerge un objeto en forma de triángulo invertido que, en este caso, se identifica como lengua. Este animal, a diferencia de los otros ya descritos, está ataviado con un casco y cinturón. Por la ligereza de su forma, parece ser más cercano estilísticamente al monumento 37 de San Lorenzo.

La segunda pieza que muestra similitud en concepto con el monumento 80 de La Venta, es la "piedra santa" del sitio Palo Gordo, Departamento de Suchitepéquez en la costa del Pacífico en Guatemala 
(Termer 1962). Esta escultura representa una figura antropomorfa sedente. A través de su trompa sostiene una serpiente en posición casi horizontal, con la cabeza de la misma hacia la izquierda de la figura. Parsons (1981: 275) sostiene que son "a pair of twisted serpents", pero la terminación izquierda de la figura viperina está destruida, lo cual no permite determinar si es otra cabeza o la cola. Se cree que las incisiones diagonales a lo largo del cuerpo de la serpiente son las decoraciones de la piel del ser viperino y no dos cuerpos de serpientes entrelazados. Esta interpretación de una sola serpiente es asimismo apoyada por el monumento 42 de Kaminaljuyú, con la cual él mismo la compara, puesto que es una sola serpiente la que cuelga del cuello de la figura $(I b i d)$.

Como comentarios finales, sólo quisiera recalcar que la mejor apreciación de los monumentos 75 y 80 de La Venta nos permite ampliar un poco más el panorama del rico mundo que nos ha legado la civilización olmeca a través de sus esculturas. La pieza completa dél monumento 75 define una clase escultórica única en el cuerpo de esculturas monumentales olmecas que, hasta lo que se conoce ahora, sólo se repite en piezas portátiles. Por otro lado, el monumento 80 representa una temática específica que se repite en otros fragmentos de esculturas olmecas de la costa del Golfo pero que, hasta recientemente, no era posible definir con claridad. Ambas piezas, dentro del género de seres sobrenaturales del corpus escultórico olmeca, definen dos diferentes sub-temas. La descripción de estas esculturas queda, en su mayor parte, en un nivel pre-iconográfico puesto que la interpretación del simbolismo específico de los elementos que las conforman aún se desconoce.

\section{BIBLIOGRAFIA}

Benson, Elizabeth P.

1981 Some Olmec Objects in the Robert Wood Bliss Collection at Dumbarton Oaks, en: The Olmec E Their Neighbors, editado por E Benson. Dumbarton Oaks Research Library and Collections, Trustees for Harvard University. Washington, D.C.

Beverido Perau, Francisco

1970 San Lorenzo Tenochtitlan y la civilización olmeca. Tesis de Maestría en Ciencias Antropológicas. Universidad Veracruzana, Xalapa. 
Castro Leal, Marcia

1982 Tigrillo olmeca de Matacanela, Veracruz. Revista Mexicana de Estudios Antropológicos, Tomo xxvir. Sociedad Mexicana de Antropología. México, D.F.

Clewlow Jr., Carl William

1974 A Stylistic and Chronological Study of Olmec Monumental Sculpture. Contributions of the University of California Archaeological Research Facility, No. 19. University of California. Berkeley.

Coe, Micheal D. y Richard A. Diehl

1980 In The Land of the Olmec. Vol. 1. University of Texas Press. Austin.

De la Fuente, Beatriz

1973 Escultura Monumental Olmeca-Catálogo. Cuaderno de Arte No. 1. Instituto de Investigaciones Estéticas. Universidad Nacional Autónoma de México, México, D.F.

1977 Los Hombres de Piedra. Escultura Olmeca. Instituto de Investigaciones Estéticas. Universidad Nacional Autónoma de México, (Segunda edición 1984) México, D.F.

González Lauck, Rebecca

1988 Proyecto Arqueológico La Venta. Arqueologia, No. 4. Dirección de Monumentos Prehispánicos. Instituto Nacional de Antropología e Historia, México, D.F.

Graham, John A.

1981 Abaj Takalik: The Olmec Style and its Antecedents in Pacific Guatemala, en: Ancient Mesoamerica, editado por J.A. Graham. A Peek Publication, Palo Alto.

1989 "Olmec Diffusion: A sculptural view from Guatemala", en: Regional Perspectives on the Olmec, editado por R. Sharer y D. Grove. 1983 School of American Research Seminar on Olmec Diffusion, Cambridge University Press, Oxford.

Joralemon, Peter David

1971 A Study in Olmec Iconography. Studies in Precolumbian Art and Ar. cheology, No. 7. Dumbarton Oaks, Trustees for Harvard University, Washington, D.C.

Medellín Zenil, Alfonso

1961 Noticias varias. Boletín del Instituto Nacional de Antropologia e Historia, No. 5 Instituto Nacional de Antıopologia e Historia, México, D.F.

1971 Monolitos Olmecas y otros en el Museo de la Universidad de Veracruz. Unión Acadèmique Internationale, Corpus Antiquitatum Americanensium, Vol. V. Instituto Nacional de Antropología e Historia. México, D.F.

Milbraith, Susan

1979 A Study of Olmec Sculptural Chronology. Studies in Pre-columbian Art $\mathcal{O}^{\circ}$ Archaeology, No. 23. Dumbarton Oaks. Trustees for Harvard University, Washington, D.C. 
Navarrete, Carlos

1974 The Olmec Rock Carvings at Pijijiapan, Chiapas, Mexico and Other Olmec Pieces from Chiapas and Guatemala. Papers of the New World Archaeological Foundation, No. 35. Brigham Young University, Provo.

Parson, Lee A.

1981 Post-Olmec Stone Sculpture: The Olmec-Izapan Transition on the Southern Pacific Coast and Highlands en: The Olmec and their Neighbors, editado por E. Benson, Dumbarton Oaks. Trustees for Harvard University, Washington, D.C.

Pohorilenko, Anatole

1977 On the Question of Olmec Deities. Journal of New World Archaeo$\log y$, Vol. II, No. 1, Instituto of Archaeology, University of California, Los Angeles.

1982 A propósito de la hacha votiva olmeca de la Casa de la Cultura de Juchitán, México. Revista Mexicana de Estudios Antropológicos, Tomo xxvir. Sociedad Mexicana de Antropología, México, D.F.

Porter, James B.

1989 Olmec colossal heads as recarved thrones: "Mutilation", revolutions and recarving. Mecanoescrito inédito. Department of Antropology, University of California, Berkeley.

Proskouriakoff, Tatiana

1979 Olmec Gods and Maya God-Glyphs. Human Mosaic. Vol. 12. DepartTermer, Franz ment of Anthropology, Tulane University, New Orleans.

1962 Apuntes arqueológicos acerca de las ruinas de Palo Gordo, Departamento de Suchitepéquez. Anales de la Sociedad de Geografia e Historia, Años xxv, Tomo xxxv, Guatemala.

\section{RECONOCIMIENTOS}

Se agradece profundamente a las siguientes personas que de una $u$ otra forma contribuyeron a la realización de este artículo: Beatriz de la Fuente, M. Judith Gallegos, John A. Graham, Lorena Mirambell, James B. Porter y el personal del iNäH e iCT del museo y zona arqueoló gica en Villa La Venta, Huimanguillo, Tabasco. 